Z Gerontol Geriat 2017 · 50:174

DOI 10.1007/s00391-017-1186-6

Online publiziert: 26. Januar 2017

(c) Springer-Verlag Berlin Heidelberg 2017

CrossMark

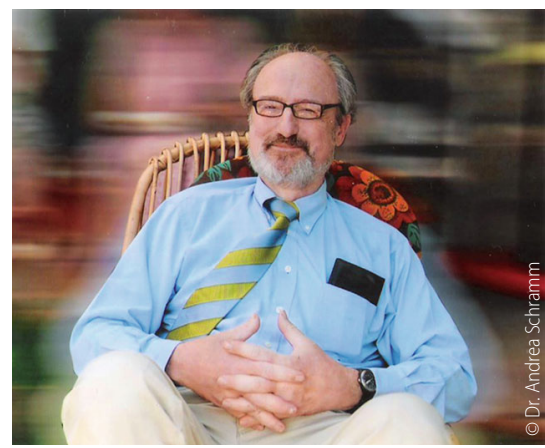

Am 23.10.2016ist Dr. med. AxelSchramm nach langer Krankheit verstorben. Dr. Schramm wurde am 06.03.1950 geboren, begann sein Medizinstudium 1969 in Hamburg und setze es 1971 in Würzburg fort. Als Medizinalassistent arbeitete er zunächst in der Anästhesie der Universität Kiel. Im Jahr 1976 promovierte er und wechselte an die Medizinische Universitätsklinik Würzburg unter Leitung von Prof. Dr. med. H. Franke, der ihn für die Zentenarenforschung begeisterte. Dr. Schramm suchte viele der damals 100-Jährigen in der Bundesrepublik persönlich auf und erhob dabei umfangreiches Datenmaterial, das auch Grundlage seiner über 100 Publikationen war. Dr. Schramm erlangte 1982 die Anerkennung als Internist; ein Jahr später wurde er zudem Kardiologe und Angiologe. Darüber hinaus erwarb er die fakultative Weiterbildung klinische Geriatrie und die Zusatzbezeichnung physikalische Therapie. Von 1983 bis 1986 war er als Oberarzt der medizinisch-geriatrischen Kliniken der RuhrUniversität Bochum im Marienhospital Herne tätig. Am 01.06.1986 zog es ihn an das Klinikum Bayreuth, wo er die medizinische Leitung der neu errichteten geriatrischen Klinik und der physikalischen Therapie übernahm. Seit 1995 war er zusätzlich Chefarzt der geriatri-

Jens Trögner

Klinikum Amberg, Amberg, Deutschland

\title{
Zum Tode von Dr. med. Axel Schramm
}

schen Rehabilitation im benachbarten Rehazentrum Roter Hügel.

Dr. Schramm hat sich zeitlebens für die Weiterentwicklung der Geriatrie engagiert. Als erster Chefarzt einer geriatrischen Krankenhausabteilung in Bayern war er Pionier einer Entwicklung, die erst Jahrzehnte später durch das Fachprogramm Akutgeriatrie eine inzwischen flächendeckende Ausweitung in Bayern findet. Dr. Schramm war Gründungsmitglied der Deutschen Gesellschaft für Geriatrie und der Ärztlichen Arbeitsgemeinschaft zur Förderung der Geriatrie in Bayern (AFGiB). Als langjähriger Vorsitzender der AFGiB und später als deren Ehrenvorsitzender hat er ganz wesentliche Impulse zur Entwicklung der Geriatrie in Deutschland und in Bayern gesetzt. Als aktives Mitglied vieler Fachgesellschaften und Fachgremien war er als Diskussionspartner sehr geschätzt. Im Jahr 2010 wurde ihm für seine Verdienste um die Geriatrie die Verdienstmedaille des Verdienstordens der Bundesrepublik Deutschland verliehen.

Dr. Schramm hat in unzähligen Veranstaltungen sein Wissen an Ärzte und nichtärztliche Mitglieder des geriatrischen Behandlungsteams weitergegeben. Er war u. a. Herausgeber des Kompendium der Gerontologie und begründete die überregional bedeutsame Fortbildungsreihe Aktuelle Geriatrie Bayreuth. Im Jahr 2007 wurden seine besonderen Verdienste um die ärztliche Fortbildung mit der Ernst-von-Bergmann-Plakette ausgezeichnet.

Dr. Schramm bleibt in Erinnerung als ein vom inneren Feuer für die Geriatrie Beseelter. Er war Ideengeber und Motor vieler wichtiger Projekte und Aktivitäten. Sein äußerst klarer und analytischer Geist war gepaart mit einer großen Por- tion Humor und Selbstironie. Dadurch wirkte er nie dogmatisch und schuf sich viele Freunde im gesamten Umfeld der Geriatrie. Dr. Schramm war über das medizinische Wissen und Können hinaus ein umfassend gebildeter Mensch mit mannigfachen Interessen. Besondere Leidenschaften galten der Musik und der Jagd.

Seine schwere Erkrankung zwang ihn 2009, die Berufstätigkeit aufzugeben. Entgegen aller Prognosen haben ihm der medizinische Fortschritt und sein beharrlicher Kampf noch einige Lebensjahre geschenkt, auch wenn zeitweise gravierende gesundheitliche Beeinträchtigungen hinzunehmen waren. Bis kurz vor seinem Tod hat er trotz seiner schweren Erkrankung an Diskussionen und Veranstaltungen aktiv teilgenommen. Sein Rat war dabei stets willkommen. Zuletzt war die Krankheit jedoch stärker als die verbliebenen Kräfte. Ihm, der sich besonders für die Erforschung der Hochaltrigkeit und die Verbesserung der Versorgung dieser Patientengruppe einsetzte, war kein langes Leben gegeben. Im Alter von 66 Jahren verstarb Dr. Schramm. Die Geriatrie in Deutschland und speziell in Bayern verliert mit ihm einen allseits respektierten und geschätzten ärztlichen Kollegen und einen Pionier seines Fachgebiets. Sein Tod hinterlässt eine schmerzliche Lücke v. a. für seine Familie und auch für die Geriatrie in Deutschland.

\section{Korrespondenzadresse}

\section{Dr. J. Trögner}

Klinikum Amberg

Mariahilfbergweg 7, 92224 Amberg,

Deutschland

troegner.jens@klinikum-Amberg.de 